\title{
Queratitis por Pseudomonas aeruginosa asociada al uso de lentes de contacto de hidrogel de silicona de última generación: Reporte de un caso
}

\author{
Edgar Delgado C., Patricia Durán O., Orlando Neira S. y Claudia Veloza G.
}

\section{Pseudomonas aeruginosa keratitis associated with the use of last generation contact lens made of silicone hydrogel: case report}

We report the case of a female patient, 56-year-old housewife, for the first time user of last generation contact lenses: Lotrafilcon B, which presented a severe corneal ulcer by Pseudomonas aeruginosa in hev left eye and subsequently required keratoplasty. Initially she reported pain and arrived at the emergency department with red eye, corneal central ulcer of three days of evolution and hypopion. Initially she received topic mydriatic drugs and prednisolone at 1\%. At the next day the ophthalmologycal exam showed hypopion at $5 \%$ and a central severe ulcer greater than $3 \mathrm{~mm}$ in diameter with sharp edges and mucopurulent secretion. The treatment was changed to moxifloxacin and natamycin. The microbiological analysis performed in two laboratories yielded Aspergillus sp. and Pseudomonas aeruginosa sensitive to ciprofloxacin, tobramycin, gentamicin and moxifloxacin. The presence of Aspergillus was interpreted as a pollution lens case and likely colonization of the cornea because of the patient good performance. After four months although improving she required corneal transplantation. Photographic documentation of the case under illumination with slit lamp is presented.

Key words: Pseudomonas aeruginosa, corneal ulcer, contact lenses.

Palabras clave: Pseudomonas aeruginosa, úlcera corneal, lentes de contacto.

\section{Introducción}

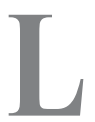

a queratitis microbiana (QM) es una seria complicación asociada al mal uso de lentes de contacto (LC) y puede presentarse con cualquier tipo de LC. Holden y cols ${ }^{1}$, estimaron que la incidencia de QM en pacientes que usaban lentes de hidrogel de silicona en porte prolongado era de 0,53 casos anuales por cada 10.000 pacientes. Este valor es 40 veces menor que el reportado para uso prolongado y ocho veces menor que el uso diario de lentes de hidrogel ${ }^{2}$. Los estudios se realizaron in vitro, con materiales como Tefilcon, Bufilcon y Perfilcon. Sin embargo, se requieren nuevos estudios con materiales de última generación como Lotrafilcon A y B.

La adherencia de Pseudomonas sp a los lentes de contacto ha sido estudiada por muchos autores. Miller y Ahearn (1987) determinaron que las proteínas lagrimales como albúmina, gammaglobulinas y lisozimas no son eliminadas completamente con la solución de mantenimiento ${ }^{3}$, causando que estas proteínas se adhieran a la superficie del lente, facilitando la adherencia de Pseudomonas sp, más aún si éste se encuentra rayado. La virulencia de Pseudomonas aeruginosa en la QM, se ha asociado posteriormente a varios factores extracelulares y productos bacterianos como son proteasas alcalinas, proteasa IV, exotoxina A, exo-enzima B y a una reciente proteasa pequeña, denominada PASP (P. aeruginosa small protease) que contribuye a las erosiones epiteliales en la queratitis causada por este agente ${ }^{4}$. Recientemente, un grupo de moléculas bacterianas señalizadoras, conocidas como N-acilhomoserina lactonas (AHLs), se han reportado también como factores importantes en la virulencia de la misma. La correlación existente entre estos factores de virulencia y adherencia ${ }^{5}$, como la producción de señales moleculares y la expresión de características fenotípicas, incluyendo la producción de enzimas, se ha estudiado por cromatografía y bioensayos en los últimos años. Por otra parte, la falta de lágrima que se produce por baja lubricación en el uso extendido, es un factor que debería tenerse en cuenta en los usuarios de lentes de contacto, debido a que la lágrima es la portadora de lisozimas, lactoferrinas, $\beta$-lisinas e inmunoglobulinas que al estar el lente deshidratado, facilita la adherencia molecular de $P$. aeruginosa. En mayo de
Hospital Militar Central, Bogotá, Colombia (EDC) Fundación Universitaria del Área Andina, Pereira, Colombia (PDO, ONS, CVG)

Recibido: 24 julio 2007 Aceptado: 15 mayo 2008

\section{Correspondencia a:}

Patricia Durán Ospina pduran@funandi.edu.co 
2007 investigadores de la Escuela de Optometría de la Universidad de Berkeley demostraron en un modelo experimental la protección del fluido lagrimal contra la queratitis por Pseudomonas $\mathrm{sp}^{6}$. Previamente estos autores habían comunicado la adherencia de esta bacteria en baja lubricación ocular en humanos.

Los factores que predisponen a una invasión patógena corneal son principalmente la exposición al mismo, la adherencia a la córnea y la invasión al estroma corneal.

Hipoxia. El hecho de que la córnea y su integridad estructural permanezcan en condiciones de transparencia y avascular es el principal criterio por el cual los profesionales pueden juzgar la seguridad del uso de LC. Es mucho lo que se ha escrito sobre las necesidades de la córnea por una adecuada oxigenación cuando se usan LC (EOP-equivalent oxygen percentage), como también de la habilidad de gran cantidad de materiales para LC para proporcionar dicha oxigenación $(\mathrm{Dk}, \mathrm{Dk} / \mathrm{t})^{8}$. Nuestro conocimiento actual sobre la hipoxia asociada al uso de LC ha llevado al desarrollo de materiales excepcionales con alto $\mathrm{Dk}$, tanto en polímeros RGP como en polímeros de hidrogel de silicona, los cuales pueden mantener la córnea libre de la hipoxia inducida por el uso de los LC, en condiciones de uso diario y de uso continuo.

En 1980, Mertz ${ }^{9}$, describió que 4\% de edema era la respuesta corneal fisiológica en condiciones de ojo cerrado durante el sueño. En 1984 Holden y Mertz ${ }^{10}$ fueron los primeros en estimar los requerimientos mínimos de transmisibilidad (Dk/t) de oxígeno que un LC debe proporcionar para que en, condiciones de uso de párpados cerrados durante el sueño, el edema corneal resultante se limite únicamente a dicho $4 \%$ de edema fisiológico; el dato estimado fue de 87 barrers [barrer es la unidad de transmisión de oxígeno. Un barrer $=1 \mathrm{X}$ $10^{9}\left(\mathrm{~cm} \mathrm{x} \mathrm{ml} \mathrm{O}_{2}\right) / \mathrm{s} x \mathrm{ml}$ x mmHg]. En 1988, La Hood y cols ${ }^{11}$ redefinieron el nivel de edema corneal fisiológico durante el sueño en 3,2\%. Lo anterior llevó a que posteriormente fuera establecido en 89 barrers el valor de $\mathrm{Dk} / \mathrm{t}$, de manera independiente por Harvitt $\mathrm{y}$ Bonanno $^{12}$ en 1999 y esto llevó a determinar que los requerimientos mínimos de oxígeno para un uso extendido seguro de los LC sea de 125 barrers (89 barrers para evitar hipoxia a nivel epitelial y 125 barrers para evitar hipoxia en todas las capas corneales).

De manera paralela, diversos estudios han demostrado el papel de la hipoxia en la unión de Pseudomonas sp a las células del epitelio corneal, requisito indispensable para que esta bacteria infecte la córnea ${ }^{13}$. Cavanagh $^{14}$ afirma que para evitar el incremento de adhesión de $P$. aeruginosa a las células epiteliales de la córnea, un lente de contacto debe tener un Dk/t mayor a 125 , independiente del tipo de lente y de la forma como el paciente use el lente, ya sea en uso diario o en uso continuo. Si los pacientes duermen con lentes de contacto se aumenta el riesgo de padecer esta patología $^{15,16}$. La adherencia de Pseudomonas sp al epitelio corneal ha sido ampliamente estudiada en usuarios de lentes de hidrogel de silicona de alto Dk, utilizados en condiciones de uso continuo ${ }^{17}$. Aunque se han asociado numerosos microorganismos con el desarrollo de la QM, la mayoría de las infecciones en usuarios de uso extendido, tanto con hidrogeles como con hidrogeles de silicona, se describen causadas por Pseudomonas sp., especialmente la especie aeruginosa.

Los infiltrados estromales en la QM por Pseudomonas sp son densos, acompañados de edema grave, gran excavación epitelial central y descarga mucopurulenta adherida a la lesión. Puede también haber un infiltrado en forma de anillo debido a la liberación de endotoxina ${ }^{18}$ y la necrosis estromal se presenta debido a la liberación de exotoxinas, endotoxinas y numerosas enzimas proteolíticas (Gráfico 1).

Los pacientes que presentan signos y síntomas consistentes con una QM requieren un manejo médico inmediato. Las úlceras pequeñas $(<2 \mathrm{~mm})$ en el estroma anterior usualmente responden bien a la monoterapia con fluoroquinolonas, prescritas agresivamente de acuerdo a los protocolos establecidos. Las úlceras mayores ( $>2 \mathrm{~mm}$ ) y más graves, y aquellas que se encuentran en el eje visual, deben tratarse con fluoroquinolonas tópicas en combinación con antimicrobianos reforzados. Los ciclopléjicos se utilizan para mitigar la reacción de la cámara anterior, al igual que para prevenir sinequias posteriores.

Habitualmente no se hacen cultivos en úlceras periféricas pequeñas, en cambio deben hacerse en lesiones mayores de $2 \mathrm{~mm}$, o que comprometan más de un tercio del espesor corneal o el eje visual. El cultivo debe incluir además de la secreción, el estuche del lente y la solución, como ocurrió en el caso que presentamos a continuación.

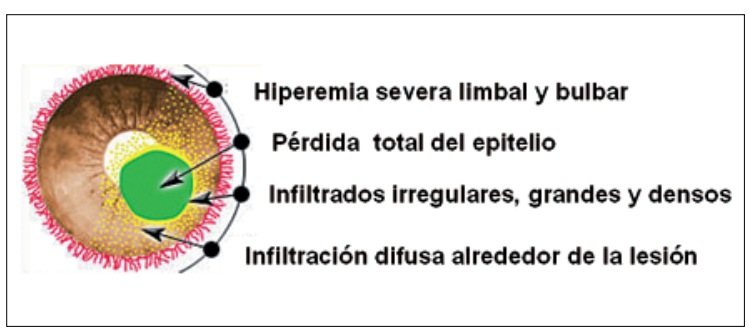

Gráfico 1. Esquema de los signos presentados en queratitis microbiana (MK) 


\section{Diagnóstico diferencial}

Las características de la QM que clínicamente la diferencian de otros eventos infiltrativos asociados al uso de LC son: aumento en la gravedad de los signos y síntomas después de descontinuar el uso de los LC, infiltrados irregulares con bordes elevados, lesiones satélites, secreción mucopurulenta adherida a la lesión, edema palpebral, hiperemia bulbar y limbar difusa grave, reacción endotelial y marcada reacción de la cámara anterior (posible hipopión, destellos y células).

\section{Caso clínico}

Paciente de 56 años, ama de casa, pedicurista y manicurista, sin antecedentes de enfermedades sistémicas u oculares, usuaria por primera vez de LC lotrafilcon $\mathrm{B}$ en período de adaptación de tres meses, sin inconvenientes. Dormía con los lentes semanalmente y los retiraba en los fines de semana para limpieza. No había sentido molestias oculares y le faltaba una semana para el cambio al nuevo par de lentes. En la noche anterior a la consulta presentó intenso dolor, fotofobia y blefarospasmo.

Al momento del ingreso presentaba una úlcera corneal grave en su ojo izquierdo (Figura 1).

$\mathrm{Al}$ abrir el estuche al ingreso, se encontró un punto negro, visible a simple vista, el cual correspondió a micelio con hifas aseptadas y detritos, a la observación microscópica (40X) con coloración directa con azul de lactofenol (Figura 2).

La superficie del lente, observada al ingreso, al microscopio, demostró estar rayada y en mal estado (Figura 3).

En el control oftalmológico se constató hiperemia conjuntival asociada a una lesión central corneal de bordes regulares, con 6 milímetros de diámetro, e hipopión. No había infiltrados satélites, ni anillo periquerático. Abundante secreción purulenta en el saco conjuntival. La paciente tenía tatuaje permanente como se observa en la foto (Figura 4).

Para el análisis microbiológico, el estuche, el lente de contacto y la solución multipropósito fueron sembrados en agar Saboureaud a $22^{\circ} \mathrm{C}$ e incubados durante ocho días, agar manitol para Staphylococcus sp durante 24 horas a $37{ }^{\circ} \mathrm{C}$ y agar cetrimida para intentar aislamiento de Pseudomonas sp. Tras cuatro días de incubación, se obtuvo crecimiento de Aspergillus sp. en el agar Saboureaud a partir del estuche del lente, y la solución multipropósito. El crecimiento fue negativo para Staphylococcus sp y positivo en agar cetrimida para $P$. aeruginosa, siendo ésta última sensible a ciprofloxacina, tobramicina, gentamicina, moxifloxacina

en el antibiograma por difusión. Se enviaron las muestras a otro laboratorio confirmándose el mismo resultado. La paciente se controló hasta observar ausencia de hipopión. Posteriormente, en ausencia de Pseudomonas sp, se apreció a la paciente asintomática, con pupila reactiva, evidenciándose cierre central en forma lenta. Se indicó diclofenaco, flurometalona y atropina al 1\%. Al día 18 la paciente no presentaba fotofobia, pero se observó un leucoma residual central. Al tercer mes de tratamiento, se remitió para queratoplastía, la cual se realizó a los seis meses de evolución.

La presencia de Aspergillus sp que se reportó en el estuche y en el lente de contacto, hizo plantear la colonización de la córnea. Sin embargo, por la evolución clínica, la ausencia del hongo en el cultivo de la córnea y quizás por su tratamiento inmediato con natamicina, se logró detener una infección que tuvo el riesgo convertirse en endoftalmitis. Es bien sabido que Pseudomonas sp abre el epitelio corneal permitiendo la entrada del hongo.

A los seis meses de evolución y tras la consecución de un donante la paciente fue programada para queratoplastía.

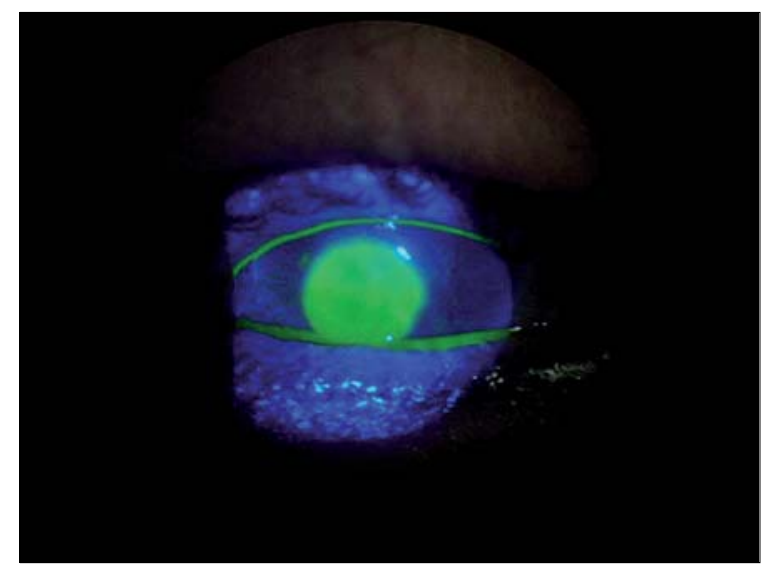

Figura 1. Fotografía bajo lámpara de hendidura con fluoresceína tomada tras seis días de tratamiento con moxifloxacina. Obsérvese el tamaño de la lesión y la ubicación central de la lesión.

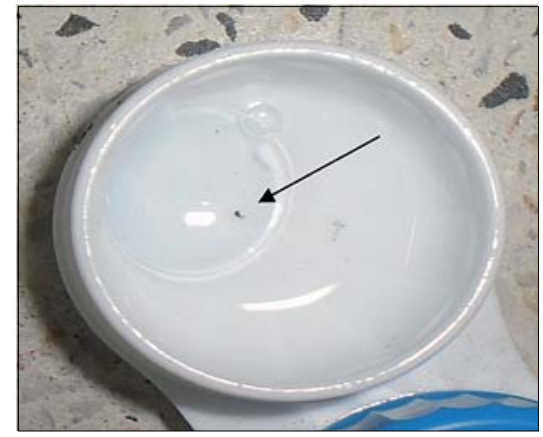

Figura 2. Estuche del lente del O.I. con punto negro.

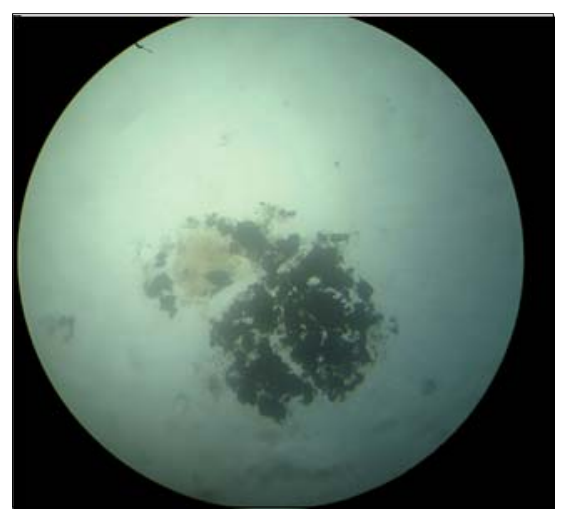

Figura 3. Fotografía del micelio de Aspergillussp con microscopio bajo aumento de 40X. Previo a aislamiento en agar Sabouroud. 
Figura 4. Estado del lente de contacto bajo microscopia directa en 40X.
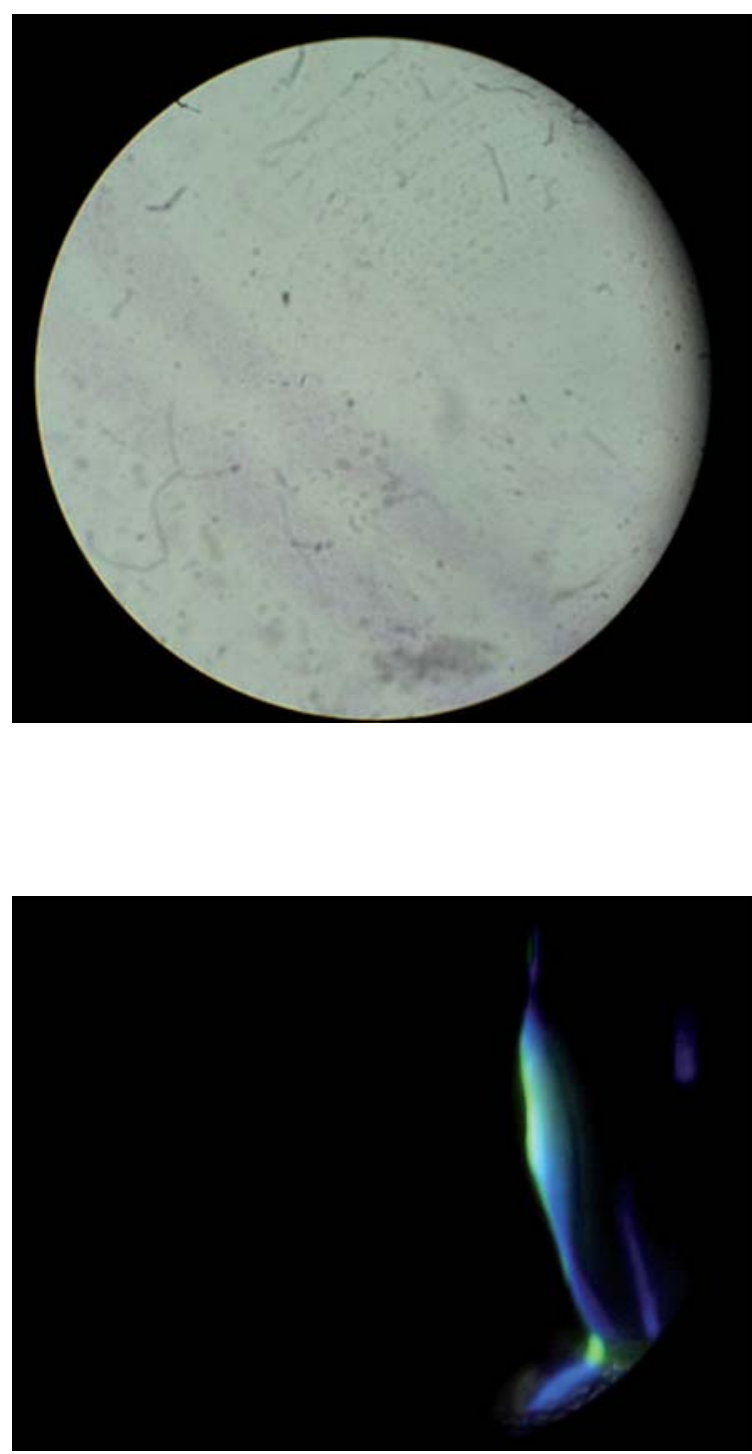

Figura 6. Examen bajo lámpara de hendidura con iluminación tangencial invertida. Se observa el perfil y la profundidad de la lesión.

Figura 7. Fotografía con fluoresceína bajo lámpara de hendidura tras dos meses de evolución.

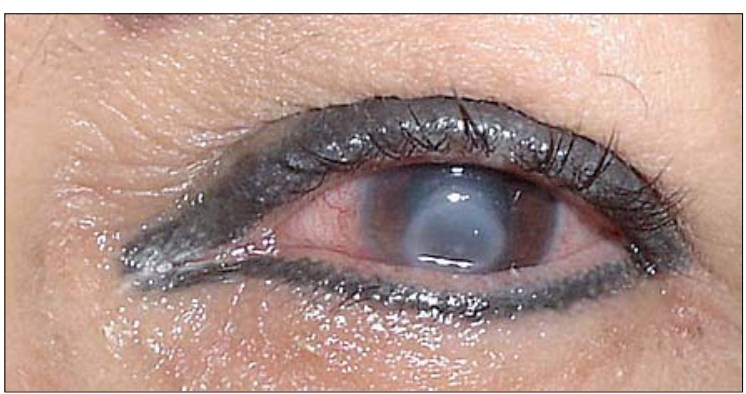

Figura 5. Queratitis por Pseudomonas aeruginosa con seis días de evolución. Nótese la secreción purulenta en canto interno. La paciente tenía blefaropigmentación cosmética.

\section{Discusión}

El caso presentado no es común en la práctica de los contactólogos y constituye un desafío interdisciplinario, frente al cual, se debe actuar de inmediato, aplicando las medidas necesarias para salvar el ojo frente a la agresividad de un patógeno, incluyendo el diagnóstico microbiológico. El entrenamiento de médicos generales en los servicios de urgencia para el manejo de este tipo de lesión debe ser una responsabilidad de los especialistas en salud visual. Se debe descartar, como diagnóstico diferencial, Acanthamoeba sp en presencia de anillo estromal en el limbo, intenso dolor y pseudodendritas, o queratitis fúngica que manifiesta infiltrados satélites de aparición más lenta y bordes muy irregulares en la lesión. Una buena anamnesis es muy útil para descartar que el paciente haya empleado agua de la llave, lo cual se asocia frecuentemente a infección por Acanthamoeba sp en usuarios de lentes de contacto. Es importante registrar la fecha de aparición, el tamaño, la profundidad y la ubicación central o periférica de la lesión, pues la córnea es más vulnerable inmunológicamente al centro que en la periferia. Mientras se obtienen los reportes microbiológicos, se debe realizar un seguimiento diario al paciente hasta haber controlado el agente etiológico.

Los pacientes que presentan signos y síntomas consistentes con una QM requieren un manejo médico inmediato. Las úlceras pequeñas $(<2 \mathrm{~mm})$ en el estroma anterior usualmente responden bien a la monoterapia con fluoroquinolonas, prescritas agresivamente, de acuerdo a los protocolos establecidos. Las úlceras mayores ( $>2 \mathrm{~mm}$ ) y más graves, y aquellas que se encuentran en el eje visual, deben mejor tratarse con fluoroquinolonas tópicas: ciprofloxacina, moxifloxacina o gatifloxacina. Los ciclopléjicos se utilizan para mitigar la reacción de la cámara anterior, al igual que para prevenir sinequias posteriores. 
Aunque habitualmente no se hacen cultivos en presencia de úlceras periféricas pequeñas, éstos deben hacerse cada vez que se observen lesiones mayores de $2 \mathrm{~mm}$, o que comprometan más de un tercio del espesor corneal, o que estén en el eje visual, o que sean pacientes pertenecientes a "grupos de riesgo" (ancianos, inmunocomprometidos, o monoculares) ${ }^{18}$.

Los cultivos deben también hacerse a las soluciones que usa el paciente para el cuidado de los LC, siempre que se pueda, para aumentar la probabilidad de obtener una identificación positiva del patógeno. Los casos graves requieren de la derivación a un especialista en córnea. Un tratamiento agresivo y precoz de la QM puede llevar a un buen reestablecimiento de la visión, aunque quedará una cicatriz permanente.

Pseudomonas aeruginosa es muy agresiva ya que genéticamente está dotada de varios mecanismos de adherencia a diferentes superficies, incluso a los materiales más tecnificados de lentes de contacto. Además se ha reportado que modifica la mucina lagrimal ${ }^{19}$. Al mismo tiempo, una gran cantidad de evidencia ha demostrado los efectos adversos de la hipoxia corneal en casi todas las capas de la córnea. Los estudios muestran una reducción en la adhesión intercelular de las células epiteliales, como también un incremento en los niveles de adherencia de $P$. aeruginosa a las células superficiales corneales exfoliadas en usuarios de lentes de hydrogel de bajo $\mathrm{Dk}^{20}$. Los estudios realizados con usuarios de LC de hydrogel de silicona de alto Dk, utilizados en condiciones de uso continuo, han mostrado niveles reducidos de adherencia de Pseudomonas $^{16-21}$.

Los LC que eliminan la hipoxia y reducen la adhesión bacteriana conllevan a un epitelio más saludable. Los LC de hydrogel de silicona no eliminan la posibilidad de una QM pero es muy probable que reduzcan enormemente su incidencia, quizá a aquellos bajísimos niveles que presentan los usuarios de hidrogeles en condiciones de uso diario ${ }^{21}$.

Un régimen de lubricación más riguroso en los usuarios de estos lentes prevendría estas infecciones graves causadas por Pseudomonas sp que ponen en riesgo la integridad visual.

El uso de lentes de contacto es un factor de riesgo para la QM en general $^{21}$ si no se realiza una correcta higiene y se utiliza por más tiempo del permitido, pues se induce a hipoxia y deshidratación corneal. Debe evaluarse a la vez, la frecuencia de uso de lubricante ocular, evaporación lagrimal y alteración en la PLPL (película lipídica prelente).

Un punto clave para comprender la fisiopatología de la infección por Pseudomonas sp es saber por qué esta bacteria no se adhiere a la córnea sana, cuando se sabe que sí lo hace a la mayoría de las superficies, incluso inertes, sin necesidad de receptores específicos. La respuesta se halla en las capas protectoras naturales de la superficie corneal, concretamente en la mucina de la película lagrimal y el glicocálix de la superficie epitelial (que también contiene moléculas de mucina y fibronectina); estas estructuras inhiben la adherencia de Pseudomonas a la superficie intacta de la córnea sana. El mecanismo preciso consiste en que la unión de Pseudomonas a las moléculas de mucina inhibe competitivamente la adherencia bacteriana a la córnea. El porte de LC elimina estas capas protectoras y permite la adhesión de la bacteria al epitelio.

\section{Recomendaciones}

Es importante, para prevenir estos casos, que el paciente tenga lubricado su lente de contacto diariamente, pues la resequedad ocular aumenta la adherencia de los patógenos a la superficie corneal. La lágrima es una barrera inmunológica que debe ser sustituida en los usuarios de LC con mayor frecuencia de uso. Es necesaria una muy buena higiene semanal, no sólo con la solución multipropósito, sino incluso con un limpiador que retire detritos y proteínas. Los autores creen que el tatuaje ocular previo, puede favorecer el desequilibrio de la microbiota ocular normal y predisponer a la presencia de patógenos más agresivos como Pseudomonas sp. Los casos reportados recientemente de infecciones causadas por Fusarium sp con soluciones preservadas con poliamino-propil-biguanida, nos ponen en alerta para regímenes de limpieza más agresivos contra estos patógenos muy adaptados. Por otra parte, ante la presencia de úlcera corneal central en usuarios de lentes de contacto, se debe iniciar terapia antibacteriana sin corticosteroides, preferiblemente con fluoroquinolonas, con valoración diaria, hasta eliminar el patógeno agresivo. Como una valoración previa para los pacientes, los oftalmólogos y contactólogos deberían realizar una anamnesis con un instrumento adecuado para usuarios de lentes de contacto, que permita al profesional y al paciente conocer el buen cuidado y mantenimiento de éstos, para prevenir estos casos que ponen en riesgo la salud visual.

Agradecimientos. A nuestra paciente por su cooperación durante todo el proceso. A Mónica Márquez. Optómetra. U.L.S. por su valiosa colaboración para la fotografía y la documentación del caso. A los evaluadores y colaboradores de la Revista Chilena de Infectología por sus valiosos aportes y recomendaciones para la publicación del manuscrito. 


\section{Resumen}

Se comunica el caso de una paciente mujer, de 56 años de edad, ama de casa, usuaria por primera vez de lentes de contacto de última generación: lotrafilcon $\mathrm{B}$, que presentó una úlcera corneal grave por Pseudomonas aeruginosa en su ojo izquierdo y posteriormente requirió queratoplastía. Inicialmente reportó dolor y llegó a la unidad de urgencia con ojo rojo, úlcera corneal central de tres días de evolución e hipopión. El tratamiento farmacológico inicial fue con midriático y prednisolona al $1 \%$. La valoración oftalmológica del día siguiente demostró hipopión al 5\% y úlcera central grave mayor a $3 \mathrm{~mm}$ de diámetro con bordes definidos y secreción mucopurulenta. Se cambió tratamiento a moxifloxacina y natamicina. El análisis microbiológico en dos laboratorios, confirmó el aislado de Aspergillus sp y Pseudomonas aeruginosa; esta última sensible a ciprofloxacina, tobramicina, gentamicina y moxifloxacina. La presencia de Aspergillus se interpretó como contaminación del estuche y probable colonización de la cornea por la clínica y buena evolución. A los cuatro meses de evolución, aunque presentó mejoría, requirió trasplante corneal. Se incluye documentación fotográfica del caso bajo iluminación con lámpara de hendidura.

\section{Referencias}

1.- Holden B A, Sweeney D F, Sankaridurg P R, Camt N, Edwards K, Stretton S, et al. Microbial keratitis and vision loss with contact lenses. Eye Contact Lens 2003; 29S: S131-4. discussion S143-4, S192-4 Review

2.- Poggio E C, Glynn R J, Schein O D, Seddon J M, Shannon M J, Scardino V A, et al. The incidence of ulcerative keratitis among users of daily-wear and extended wear soft contact lenses. N Engl J Med 1989; 321: 779-83.

3.- Miller M J, Ahearn D G. Adherence of Pseudomonas aeruginosa to hydrophilic contact lenses and other substrata. J Clin Microbiol 1987; 25:1392-7.

4.- Marquart M E, Caballero A R, Chomnawang $\mathrm{M} \mathrm{T}$, Brett A, Twining S S, O`Callaghan R J. Identification of a novel secreted protease from Pseudomonas aeruginosa that causes corneal erosions. Invest Ophthalmol Vis Sci 2005; 46: 3761-8

5.- Zhu H, Thuruthyil S, Wilcox M D. Determination of quorum-sensing signal molecules and virulence factors of Pseudomonas aeruginosa isolates from contact lens-induced microbial keratitis. J Med Microbiol 2002; 51: 1063-70.

6.- Kwong M, Evans D, Minjian N I, Cowell B,
Fleiszig S. Human tear fluid protects against Pseudomonas aeruginosa keratitis in a murine experimental model. Infect Immun 2007; 75: 2325-32.

7.- Hill R. The corneas need to breathe. Int Contact Lens Clin 1976; 3: 60-1.

8.- Fatt I, St Helen R. Oxygen tension under an oxygen permeable contact lens. Am J Optom Arch Am Acad Optom 1971; 48: 545-55.

9.- Mertz G. Overnight swelling of the living human cornea. J Am Optom Assoc 1980; 51: 211-4

10.- Holden B and Mertz G. Critical oxygen levels to avoid corneal edema for daily and extended wear contact lenses. Invest Ophthalmol Vis Sci 1984; 25: 1161-7.

11.- La Hood D, Sweeney D, Holden B. Overnight corneal edema with hydrogel, rigid gas-permeable and silicone elastomer contact lenses. Int Cont Lens Clin 1988; 15 149-54.

12.- Harvitt D, Bonanno J. Re-evaluation of the oxygen diffusion model for predicting minimum contact lens $\mathrm{Dk} / \mathrm{t}$ values needed to avoid corneal anoxia. Optom Vis Sci 1999; 76: $712-9$

13.- Efron N. Complicaciones de las lentes de contacto. $2^{\mathrm{a}}$ ed. 2005. ELSEVIER, Madrid, España.

14.- Ghormley N R. Qué tanto oxígeno es suficiente para usar un lente en forma segura? Contact Lens Spectrum. Marzo 2005 http://www.clspectrum.com/ article. aspx article $=12759$

15.- Chalupa E, Swarbrick H, Holden B, Sjöstrand J. Severe corneal infections associated with contact lens wear. Ophthalmology 1987; 94: 17-22.

16. - Buehler P O, Schein O D, Stamler J F, Verdier D D, Katz J. The increased risk of ulcerative keratitis among disposable soft contact lens users. Arch Ophthalmol 1992; 110: $1555-8$

17.- Ren D, Yamamoto K, Ladage P M, Molai M, Li L, Petroll W M, et al. Adaptive effects of 30-night wear of hyper$\mathrm{O} 2$ transmissible contact lenses on bacterial binding and corneal epithelium: a 1-year clinical trial. Ophthalmology 2002; 109 : 27-39; discussion: 39-40.

18.- Weissman B, Giese M, \& Mondino B. Ulcerative bacterial keratitis. In: Silbert J (ed): Anterior segment complications of contact lens wear, 2nd ed. Boston, Butterworth-Heinemann, 2000. 225-49

19.- Cavanaugh $\mathrm{H}$, Ladage $\mathrm{P}$, Li S, et al. Effects of daily and overnight wear of a novel hyper oxygen-transmissible soft contact lens on bacterial binding and corneal epithelium: a 13-month clinical trial. Ophthalmology 2002; 109: 1957-69. 